Quim. Nova, Vol. 36, No. 6, 870-873, 2013

\title{
ESTUDO COMPARATIVO ENTRE AS METODOLOGIAS ESCHKA, INFRAVERMELHO, CONDUTIVIDADE TÉRMICA (TCD) E ULTRAVIOLETA PARA A DETERMINAÇÃO DE ENXOFRE TOTAL EM COMBUSTÍVEIS SÓLIDOS
}

\author{
Pâmela Coelho Tambani*, Jorge Luis Dias dos Santos, Danilo Eiji Hirayama, Marcelo Aparecido Mendonça e Heloisa \\ Burkhardt Antonoff \\ Laboratório de Combustíveis e Lubrificantes, Instituto de Pesquisas Tecnológicas do Estado de São Paulo S.A., 05508-901 \\ São Paulo - SP, Brasil
}

Recebido em 3/7/12; aceito em 19/12/12; publicado na web em 21/3/13

\begin{abstract}
COMPARATIVE STUDY OF ESCHKA, INFRARED, THERMAL CONDUCTIVITY (TCD) AND ULTRAVIOLET METHODS OF TOTAL SULPHUR DETERMINATION IN SOLID FUELS. The oxidation of sulphur compounds upon burning emits large quantities of SOx into the atmosphere. Therefore, there is growing interest in fast and accurate methods for analyzing sulphur content in fuels. The objective of this work was to compare four different methods of total sulphur determination in solid fuels. The methods used in this work were Eschka, Infrared, Thermal Conductivity Detection (TCD) and Ultraviolet Fluorescence Detection (UV). The preliminary results showed that TCD and UV methods (nonstandard methods for solid fuels) have similar precision to the infrared method (standard method) for high-sulphur coal samples.
\end{abstract}

Keywords: solid fuel; total sulphur; analytical methodologies.

\section{INTRODUÇÃO}

Os combustíveis sólidos representam uma importante fonte energética em produção e consumo no mundo. No Brasil, por exemplo, $4,6 \%$ do consumo final de energia são providos por carvão mineral e coque, $6,6 \%$ por lenha e $11,1 \%$ por bagaço-de-cana. ${ }^{1}$ Sendo assim, para assegurar a qualidade desses materiais, é importante aprimorar as diversas técnicas de análise existentes.

Uma das análises de extrema importância nos combustíveis sólidos é a determinação do teor de enxofre total, pois os compostos de enxofre estão associados a diversos problemas, como: corrosão, chuva ácida, formação de $\mathrm{H}_{2} \mathrm{~S}$ e diminuição do poder calorífico, sendo esse último devido à energia liberada pelo enxofre ser inferior à do carbono e do hidrogênio.

O enxofre é o heteroátomo mais abundante na composição do carvão, podendo representar de 0,1 a $6 \%$ de sua composição..$^{2-4}$ Nas biomassas, ele está presente em menor proporção.

Devido aos problemas citados, a necessidade de desenvolvimento de métodos rápidos e mais precisos para as análises de rotina de teor de enxofre tem aumentado. ${ }^{5} \mathrm{O}$ enxofre foi primeiramente determinado pelo método Eschka (ASTM D 3177, Standard Test Methods for Total Sulfur in the Analysis Sample of Coal and Coke), ${ }^{6}$ baseado na extração quantitativa do sulfato solúvel em água após a amostra ser incinerada e oxidada a $800^{\circ} \mathrm{C}$, na presença de uma mistura de óxido de magnésio e carbonato de sódio. ${ }^{7}$ Depois, outras metodologias foram introduzidas, como a detecção no Infravermelho (IV) (ASTM 4239 - Standard Test Methods for Sulfur in the Analysis Sample of Coal and Coke Using High-Temperature Tube Furnace Combustion Methods). ${ }^{8}$ Nesse método, a combinação da elevada temperatura do forno $\left(1350^{\circ} \mathrm{C}\right)$ com uma vazão de oxigênio, resultam na combustão de uma amostra de massa conhecida. Os óxidos de enxofre formados durante a combustão são coletados e mensurados pela célula de infravermelho que converte o $\mathrm{SO}_{\mathrm{x}}$ para \% de enxofre.

A quantificação do enxofre também pode ser realizada por Detector de Condutividade Térmica (TCD) e Ultravioleta (UV).

*e-mail: ptambani@ipt.br
$\mathrm{Na}$ análise por TCD a amostra é incinerada e oxidada a $1150^{\circ} \mathrm{C}$ e a detecção é realizada por células de condutividade térmica. No ensaio por UV, a amostra é queimada na presença de excesso de oxigênio, gerando radicais $\mathrm{SO}_{2}$, que fluorescem na região do ultravioleta. Como ainda não existem normas para a determinação de enxofre através desses métodos, os mesmos precisam ser validados.

O objetivo do presente trabalho é comparar os métodos de quantificação de enxofre normalizados (Eschka e infravermelho) com os não normalizados (Ultravioleta e TCD) para combustíveis sólidos, utilizando amostras de carvão com teores de enxofre de, aproximadamente, 1 a 3,5\% e uma amostra de feno de braquiária (biomassa). Com este estudo espera-se poder avaliar a viabilidade de utilização dos métodos UV e TCD para quantificação de enxofre.

\section{PARTE EXPERIMENTAL}

Os ensaios foram realizados no Laboratório de Combustíveis e Lubrificantes (LCL) do Instituto de Pesquisas Tecnológicas do Estado de São Paulo S/A - IPT.

\section{Materiais}

As amostras analisadas estão descritas na Tabela 1.

Tabela 1. Descrição das amostras

\begin{tabular}{ll}
\hline Amostra & Descrição \\
\hline Biomassa & Feno de braquiária \\
Carvão 1 & Carvão com baixo teor de enxofre \\
Carvão 2 & $\begin{array}{l}\text { Material de referência de enxofre em carvão com teor de } \\
\text { enxofre de 2,92\% }\end{array}$ \\
Carvão 3 & Carvão com alto teor de enxofre \\
\hline
\end{tabular}

Como os ensaios foram realizados em base seca, as amostras de carvão foram secas em estufa por 2 horas a $105^{\circ} \mathrm{C}$ e a amostra de biomassa conforme a norma NBR $8112 .{ }^{9}$

Todas as amostras foram analisadas em triplicata, sendo utilizada 
uma nova alíquota para cada determinação. A média das três determinações foi adotada como medida de comparação entre as diferentes metodologias.

\section{Métodos}

Os métodos utilizados no estudo de comparação foram:

\section{Determinação de enxofre total pelo método Eschka}

Para as amostras de carvão o ensaio foi realizado segundo a norma ASTM D 3177. Como não há uma norma específica para biomassa, as análises na amostra de feno de braquiária também foram baseadas nessa norma. Para a quantificação do enxofre por este método é utilizado $1 \mathrm{~g}$ de amostra.

Determinação de enxofre total com detecção no infravermelho - IV

Para as amostras de carvão o ensaio foi realizado segundo a norma ASTM D 4239. Como não há uma norma específica para biomassa, as análises da amostra de feno de braquiária também foram baseadas nessa norma. Neste método, são utilizados entre 0,1 e 0,25 g de amostra.

Os ensaios foram realizados no analisador de enxofre, marca LECO, modelo S-144 DR Dual Range Sulfur Analyzer e, para acelerar a queima, óxido de tungstênio foi adicionado à amostra. Foram construídas duas curvas analíticas, uma para baixos teores de enxofre e outra para teores mais elevados utilizando materiais de referência de enxofre em carvão. Os limites dessas curvas são definidos em função da área do gráfico referente ao perfil de queima. A curva analítica para teores baixos de enxofre tem como limites inferior e superior 0 e 3,08 unidades de área, respectivamente. Já a curva para teores elevados abrange de 0,66 a 14,14 unidades de área.

Determinação de enxofre total com detecção por células de condutividade - TCD

Os ensaios foram realizados segundo a norma ASTM D 5373. ${ }^{10}$

As análises foram realizadas em um equipamento da marca Elementar CHNOS, modelo Vario EL Cube. Foi utilizada a curva analítica construída pelo fabricante do equipamento, sendo dividida em dois segmentos: curva baixa (faixa: 226 a 4.099 unidades de área) e curva alta (faixa: 4.099 a 22.492 unidades de área). Essa curva foi ajustada antes das análises utilizando um material de referência (NIST SRM 2683b) com teor de enxofre de 1,955 $\pm 0,041 \%$.

A quantidade de amostra requerida para a quantificação do enxofre neste equipamento é de 5 a $10 \mathrm{mg}$.

Determinação de enxofre total com detecção por fluorescência na região do ultravioleta - UV

Os ensaios foram realizados segundo a norma ASTM D 5453. ${ }^{11}$

As análises foram realizadas no equipamento ANTEK série 9000. Foram utilizadas duas curvas analíticas, uma para a amostra de biomassa, com limites entre 0,02 e $0,05 \%$ de enxofre, e outra para as demais amostras, com limites entre 1 e $4 \%$.

Para a quantificação do enxofre por esse método são utilizados $50 \mathrm{mg}$ de amostra.

\section{Análise estatística}

As análises estatísticas dos resultados foram realizadas com auxílio do programa Minitab 15 (Statistical Software, State College, Pennsylvania, US).

\section{RESULTADOS E DISCUSSÃO}

Nas Figuras 1 a 4 são apresentados os valores médios do teor de enxofre de todas as amostras, em relação aos métodos utilizados.

É possível notar que, para a amostra de biomassa (Figura 1), o resultado obtido pelo método TCD foi discrepante e com elevado desvio padrão. O valor obtido em unidades de área foi de 274 , valor esse dentro da faixa de curva baixa (226 a 4.099 unidades de área). Entretanto o valor ficou próximo do limite inferior da curva, podendo causar imprecisão nos resultados. Outro motivo que pode ter contribuído para o aumento do desvio padrão é o fato da curva analítica de fábrica ter sido construída utilizando uma microbalança com seis

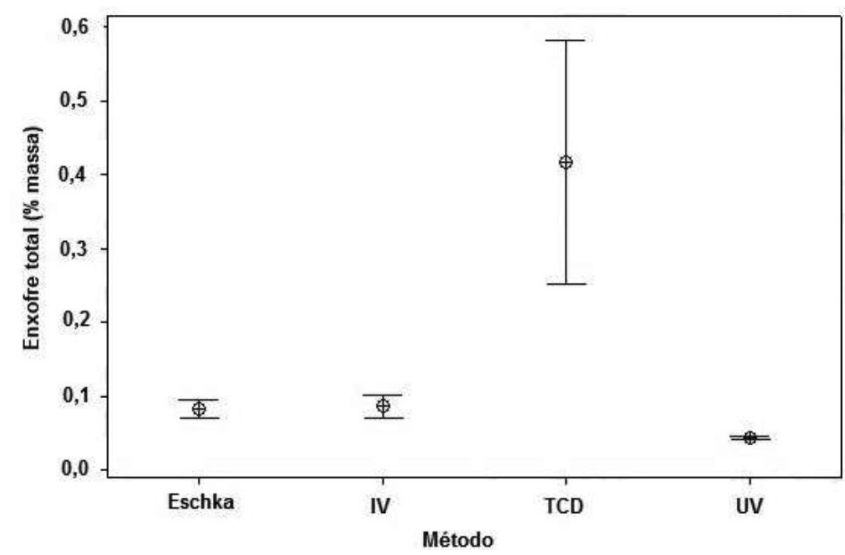

Figura 1. Valores médios e desvio padrão do teor de enxofre quantificado por diferentes metodologias para a amostra de biomassa

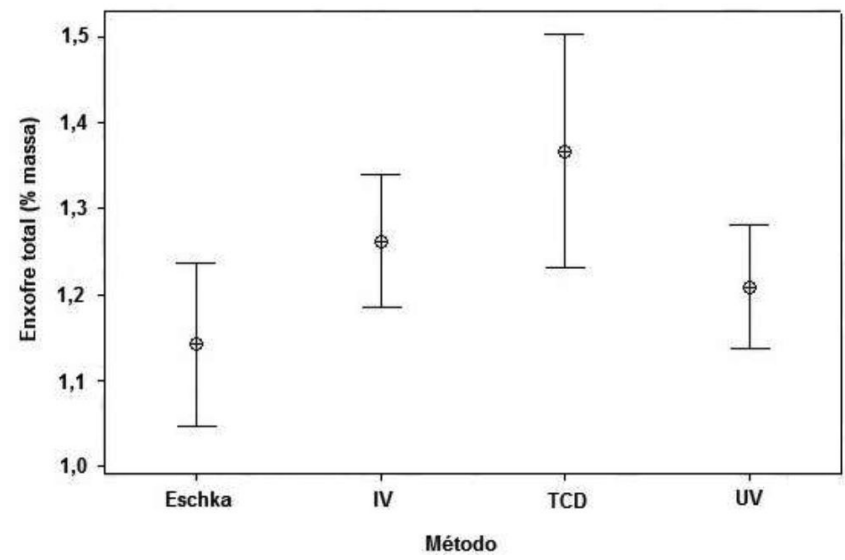

Figura 2. Valores médios e desvio padrão do teor de enxofre quantificado por diferentes metodologias para a amostra denominada carvão 1

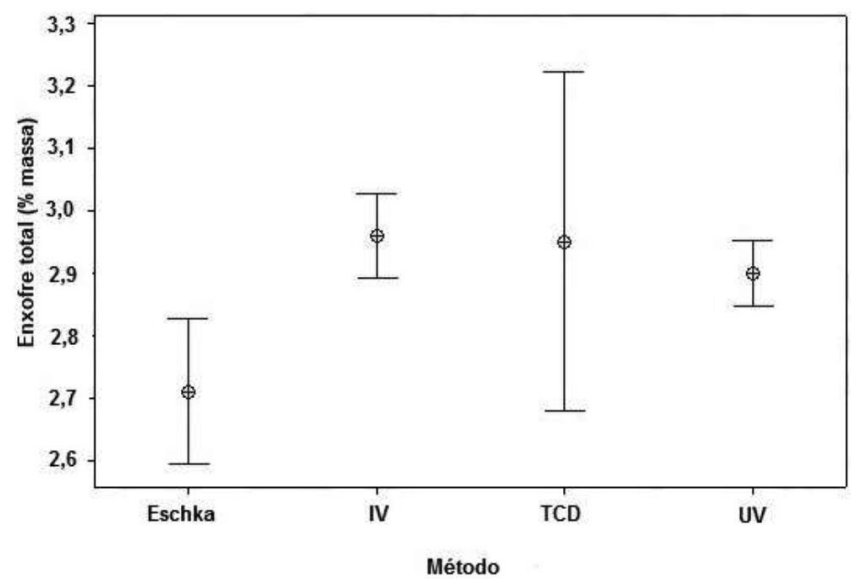

Figura 3. Valores médios e desvio padrão do teor de enxofre quantificado por diferentes metodologias para a amostra denominada como carvão 2 


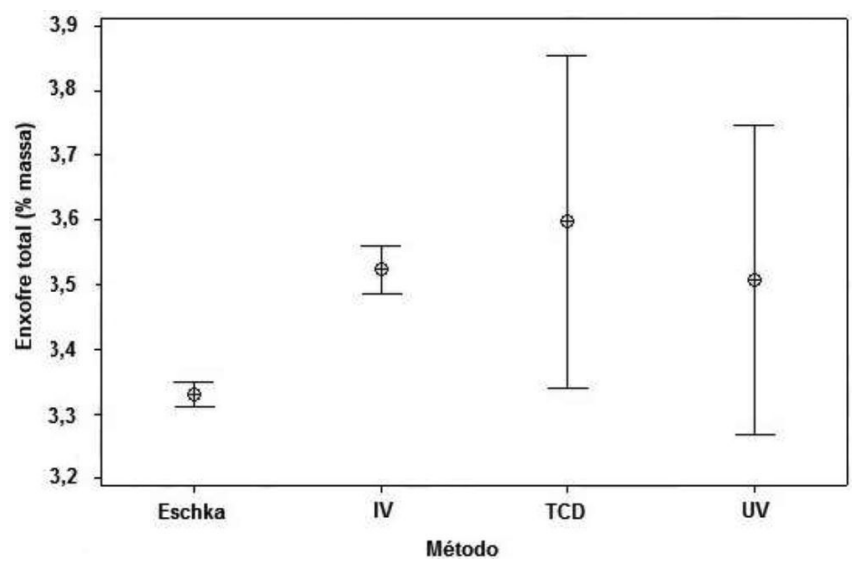

Figura 4. Valores médios e desvio padrão do teor de enxofre quantificado por diferentes metodologias para a amostra denominada carvão 3

casas decimais, enquanto as análises foram efetuadas com auxílio de uma balança analítica com precisão de $0,1 \mathrm{mg}$. Como a massa influencia diretamente no resultado, a utilização de balança com precisão menor acarreta em maiores desvios, principalmente para valores de áreas reduzidos. Também observa-se que os métodos Eschka, IV e UV apresentam resultados semelhantes para a amostra de biomassa.

Para as amostras de carvão (Figuras 2, 3 e 4), o método Eschka tende a apresentar uma média inferior às dos demais métodos e o método TCD apresenta os desvios padrões mais elevados.

Na Figura 5 pode-se comparar os valores obtidos pelas diferentes metodologias com o valor de referência da amostra carvão 2 (2,92 \pm $0,07 \%$ ). Para os métodos normalizados para quantificação de enxofre em amostras de carvão (Eschka e IV) foi utilizada a reprodutibilidade da norma na comparação do valor obtido com o valor de referência. Para o método Eschka, segundo a norma ASTM D 3177, a reprodutibilidade para teores de enxofre acima de $2 \%$ é de 0,20 . Para a quantificação por infravermelho, segundo a norma ASTM D 4239, a reprodutibilidade é dada pela seguinte equação: 0,02+0,09x, onde $\mathrm{x}$ representa o teor de enxofre obtido. Já para os métodos não normalizados (TCD e UV), foi utilizado o desvio padrão para comparação. Na Figura 5 também são mostradas duas linhas (máximo e mínimo) que representam os limites do valor de referência considerando a incerteza contida no certificado do material. Pode-se observar que o método Eschka tende a apresentar valores menores que o esperado. Essa diferença pode ser justificada pelo fato de o material de referência ter sido validado pelo método IV. Mas, considerando-se os limites de reprodutibilidade da norma ASTM D 3177 (0,20), o valor de referência está contido no intervalo dos resultados.

Para avaliar se existe diferença significativa entre as médias obtidas pelas distintas metodologias foi empregada a análise da variância (one way ANOVA). ${ }^{12}$

Anteriormente, foi realizado o teste de normalidade de AndersonDarling a um nível de confiança de 0,05. A hipótese nula nesse teste é que os resíduos apresentam uma distribuição normal. Para a amostra de biomassa o valor de " $p$ " encontrado foi inferior a 0,05 , indicando que os resíduos não tendem a uma distribuição normal ao nível de significância de 5\%. Mas, como é difícil chegar a uma conclusão sobre a normalidade, quando se utiliza um número reduzido de pontos, foi admitida distribuição normal como subjacente às populações. Já os dados experimentais referentes às amostras de carvão demonstraram uma distribuição normal, obtendo-se para a amostra de carvão 1 $\mathrm{p}=0,147$, para a amostra de carvão $2 \mathrm{p}=0,202$ e para a amostra de carvão $3 \mathrm{p}=0,452$.

Também foi realizado o teste de Levene para verificar a hipótese adotada de que as variâncias são iguais. Nesse teste a hipótese nula

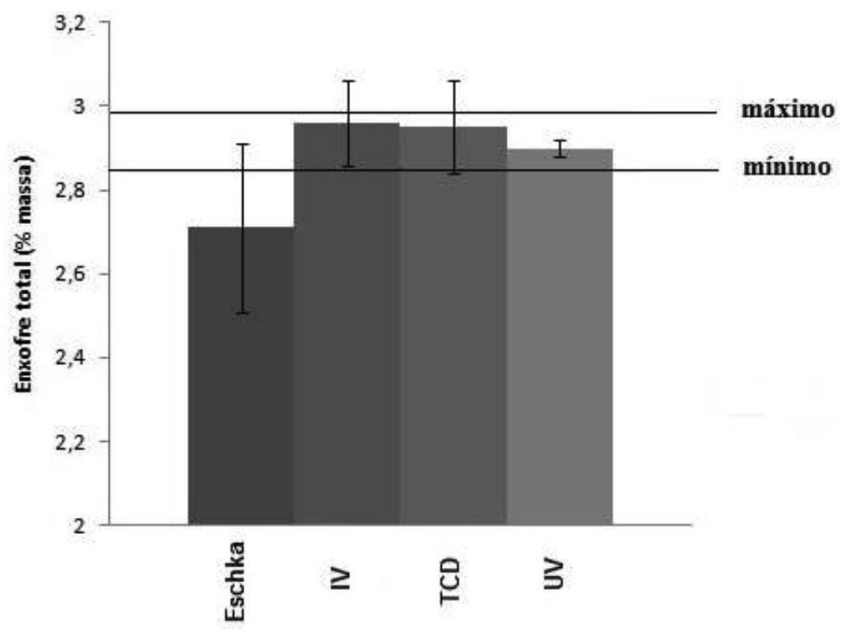

Figura 5. Comparação entre o valor de referência de teor de enxofre e os valores quantificados por diferentes metodologias para a amostra denominada carvão 2. O valor de referência está representado pelos limites mínimo e máximo, considerando o valor de referência \pm incerteza do certificado. Métodos Eschka e IV: valor médio \pm reprodutibilidade das normas; Métodos TCD e UV: valor médio \pm desvio padrão

admite que as variâncias são iguais. Na Tabela 2 são mostrados os resultados obtidos para cada amostra.

Tabela 2. Análise de igualdade de variância (Teste de Levene)

\begin{tabular}{ccccc}
\hline Amostra & Biomassa & Carvão 1 & Carvão 2 & Carvão 3 \\
\hline Valor de p & 0,230 & 0,958 & 0,330 & 0,430 \\
\hline
\end{tabular}

Como os valores de "p" encontrados foram todos maiores que 0,05, a hipótese nula é aceita, ou seja, não há diferença entre as variâncias dos grupos.

A análise da variância dos diferentes métodos de análise é apresentada na Tabela 3.

A hipótese nula testada $\left(\mathrm{H}_{0}\right)$ é que todas as médias são iguais, enquanto a hipótese alternativa $\left(\mathrm{H}_{1}\right)$ é que pelo menos uma das médias é diferente das demais. O teste foi realizado com nível de significância de $5 \%$ e os valores de "F" foram comparados. Se o F calculado é superior ao F crítico, rejeita-se a hipótese nula. Como pode-se observar na Tabela 3, os valores de "p" para todas as amostras foram menores que 0,05 , indicando que a hipótese nula deve ser rejeitada e, portanto, existe diferença entre as médias ao nível de significância de 5\%.

Como as amostras diferiram ao nível de significância de 5\%, foi realizado um método de contraste (teste de Tukey) para complementar a ANOVA e poder comparar as metodologias entre si. Os resultados obtidos estão representados na Tabela 4.

Na Tabela 4, os métodos representados pela mesma letra não apresentam diferenças ao nível de significância de 5\%. Para a amostra de biomassa, o método TCD diferiu dos demais, como já havia sido observado. Para a amostra de carvão com baixo teor de enxofre (carvão 1) apenas o método UV se equivale aos métodos Eschka e IV. Porém, não foi observada equivalência entre os métodos Eschka e IV. Nas amostras de carvão com teores mais elevados de enxofre (carvão 2 e carvão 3), os métodos IV, UV e TCD mostraram-se equivalentes. Para a amostra de carvão 3, o método Eschka também foi equivalente ao método UV.

\section{CONCLUSÃO}

Os métodos de quantificação por IV, TCD e UV se mostraram 
Tabela 3. Análise de Variância dos efeitos dos diferentes métodos de quantificação de enxofre total

\begin{tabular}{|c|c|c|c|c|c|c|}
\hline \multicolumn{7}{|c|}{ Amostra: Biomassa } \\
\hline Fonte & Graus de liberdade & Soma dos quadrados & Quadrado médio & $\mathrm{F}$ & Valor de p & Conclusão \\
\hline Efeito do tratamento & 3 & 0,2735 & 0,0912 & 80,55 & 0,000 & \\
\hline Efeito da amostra & 8 & 0,0091 & 0,0011 & & & Médias diferentes \\
\hline Total & 11 & 0,2826 & & & & \\
\hline \multicolumn{7}{|c|}{ Amostra: Carvão 1} \\
\hline Fonte & Graus de liberdade & Soma dos quadrados & Quadrado médio & $\mathrm{F}$ & Valor de $\mathrm{p}$ & Conclusão \\
\hline Efeito do tratamento & 3 & 0,0813 & 0,0271 & 17,26 & 0,001 & \\
\hline Efeito da amostra & 8 & 0,0126 & 0,0016 & & & Médias diferentes \\
\hline Total & 11 & 0,0938 & & & & \\
\hline \multicolumn{7}{|c|}{ Amostra: Carvão 2} \\
\hline Fonte & Graus de liberdade & Soma dos quadrados & Quadrado médio & $\mathrm{F}$ & Valor de $\mathrm{p}$ & Conclusão \\
\hline Efeito do tratamento & 3 & 0,1219 & 0,0406 & 10,60 & 0,004 & \\
\hline Efeito da amostra & 8 & 0,0307 & 0,0038 & & & Médias diferentes \\
\hline Total & 11 & 0,1526 & & & & \\
\hline \multicolumn{7}{|c|}{ Amostra: Carvão 3} \\
\hline Fonte & Graus de liberdade & Soma dos quadrados & Quadrado médio & $\mathrm{F}$ & Valor de $\mathrm{p}$ & Conclusão \\
\hline Efeito do tratamento & 3 & 0,1159 & 0,0387 & 7,58 & 0,01 & \\
\hline Efeito da amostra & 8 & 0,0408 & 0,0051 & & & Médias diferentes \\
\hline Total & 11 & 0,1567 & & & & \\
\hline
\end{tabular}

Tabela 4. Teste de Tukey para comparação de médias (métodos representados pela mesma letra não apresentam diferenças ao nível de significância de 5\%)

\begin{tabular}{ccccc}
\hline \multirow{2}{*}{ Metodologia } & \multicolumn{4}{c}{ Amostra } \\
\cline { 2 - 5 } & Biomassa & Carvão 1 & Carvão 2 & Carvão 3 \\
\hline Eschka & $\mathrm{a}$ & $\mathrm{a}$ & $\mathrm{a}$ & $\mathrm{a}$ \\
IV & $\mathrm{a}$ & $\mathrm{b}$ & $\mathrm{b}$ & $\mathrm{b}$ \\
TCD & $\mathrm{b}$ & $\mathrm{c}$ & $\mathrm{b}$ & $\mathrm{b}$ \\
UV & $\mathrm{a}$ & $\mathrm{a}$ b & $\mathrm{b}$ & $\mathrm{a} \mathrm{b}$ \\
\hline
\end{tabular}

equivalentes para as análises das amostras de carvão com teores de enxofre mais elevados. Para as amostras com baixo teor de enxofre será necessária a elaboração de uma curva analítica para o TCD que seja mais restrita para essa faixa de valores, além da aquisição e utilização de uma micro balança.

Quanto ao método Eschka, os resultados obtidos apresentaram uma tendência a valores menores quando comparados com os métodos IV, TCD e UV. No método gravimétrico estão envolvidas várias etapas para concluir a quantificação, resultando em 16 horas de ensaio enquanto que nos métodos automáticos esse tempo é reduzido para 10 minutos no TCD, aproximadamente 2 minutos no IV e 4 minutos no UV.

Utilizando a reprodutibilidade da norma ASTM D 3177 pode-se concluir que os resultados entre as diversas metodologias são compatíveis.

Como neste trabalho foi analisado um número reduzido de amostras e de determinações por amostras (triplicatas), as conclusões apresentadas servem como um estudo preliminar. A utilização dos métodos UV e TCD para quantificação de enxofre é viável, entretanto para a validação desses métodos será necessário novo planejamento experimental, contemplando um estudo que visa aumentar os graus de liberdade.

\section{REFERÊNCIAS}

1. https://ben.epe.gov.br/downloads/Relatorio_Final_BEN_2012.pdf, acessada em Março 2013.

2. Jim, G.; Katskov, D.; Tittarelli, P.; Talanta 2011, 83, 1687.

3. White, A.; Whittinghan, J.; Fuel 1983, 62, 1058.

4. Kasrai, M.; Brown, J.; Bancroft, G.; Yin, Z.; Tan, K.; Int. J. Coal Geol. 1996, 32, 107.

5. Santelli, R.; Oliveira, E.; Carvalho, M. F.; Bezerra, M.; Freire, A.; Spectrochim. Acta, Part B 2008, 63, 800.

6. American Society for Testing and Materials, Pennsylvania, USA, 2002; D-3177-02, Total Sulfur in the Analysis Sample of Coal and Coke.

7. Botto, R.; White, B.; Karchmer, H.; Fuel 1980, 59, 157.

8. American Society for Testing and Materials, Pennsylvania, USA, 2008; D-4239-08, Standard Test Methods for Sulfur in the Analysis Sample of Coal and Coke Using High-Temperature Tube Furnace Combustion Methods.

9. Associação Brasileira de Normas Técnicas, Rio de Janeiro, 1983; NBR8112-83, Carvão Vegetal - análise imediata.

10. American Society for Testing and Materials, Pennsylvania, USA, 2002; D-5373-02, Standard Methods for Instrumental Determination of Carbon, Hydrogen, and Nitrogen in Laboratory Samples of Coal and Coke.

11. American Society for Testing and Materials, Pennsylvania, USA, 2009; D-5453-09, Standard Methods for Sulfur in Light Hydrocarbons, Spark Ignition Engine Fuel, Diesel Engine Oil by Ultraviolet Fluorescence.

12. Box, G. E. P.; Hunter, W. G.; Hunter, J. S.; Statistics for Experimenters, an Introduction to Design, Data Analysis and Model Building; John Wiley \& Sons: New York, 1978, cap. 6. 